Lindegren, C. C. \& Lindegren, G. (1956). J. gen. Microbiol. 15, 19-28

\title{
Eight Genes Controlling the Presence or Absence of Carbohydrate Fermentation in Saccharomyces
}

\author{
BY C. C. LINDEGREN AND GERTRUDE LINDEGREN \\ Biological Research Laboratory, Southern Illinois University, \\ Carbondale, Illinois, U.S.A.
}

\begin{abstract}
SUMMARY: Genes controlling carbohydrate fermentation in Saccharomyces often control the fermentation of more than one carbohydrate. The gene MZ has at least five different manifestations involving its ability to respond to five different carbohydrate inducers for the production of a single enzyme; a series of multiple alleles of MZ differ from one another in ability to respond to the different inducers. The gene DX controls the fermentation of dextrin and glycogen. The gene ST controls the fermentation of starch; some starch-positive cultures are Schardinger-dextrinpositive. The gene SU controls the production of a constitutive enzyme which splits both sucrose and raffinose.
\end{abstract}

\section{The gene $G A$}

The Durham tube and melting-point tube methods (Lindegren, 1956) distinguish eight heritable differences in the fermentation of carbohydrates in the Carbondale breeding stock. The genes occur either in the dominant (capable of producing the enzyme) or the recessive (incapable of producing the enzyme) form. The gene-pair controlling galactose-fermentation (Lindegren \& Lindegren, 1946) was the first analysed; the dominant form is now designated by the two-letter symbol GA (previously $\mathbf{G}$ ), while the recessive is identified by ga (previously g). Because different genes may control the ability to ferment the same sugar a system for distinguishing phenotype and genotype has been introduced. The phenotypes are designated by three-letter abbreviations of the substrate fermented (the designation of the fermenter is by capitals and the non-fermenter by lower-case letters), while two-letter abbreviations describe the genotypes. GA controls the production of an adaptive enzymesystem which has the end result of isomerizing galactose into a utilizable hexose. Whether the enzyme system is the galactowaldenase of Cardini, Caputto, Paladini \& Leloir (1950) which converts galactose-1-phosphate to glucose-1-phosphate and which needs uridine diphosphoglucose (UDPG) as coenzyme or 'galactokinase' (galactose + ATP $\rightarrow$ galactose-1-phosphate) or enzymes needed for the synthesis of UDPG has not been determined. Unadapted GA cultures (and cultures carrying ga) lack the enzyme system for this fermentation. Only one gene-pair controlling the presence or absence of ability to ferment galactose has been discovered in the Carbondale stock. Cultures in the Carbondale stock which ferment galactose on full nutrient medium within 8 days in the Durham tube and within $6 \mathrm{hr}$. in the meltingpoint tube are identified as GA; any culture which fails to ferment galactose under these standard conditions is identified as ga. 
All the cultures of the Carbondale stock are able to ferment D-glucose and D-fructose; release of one of these hexoses from an oligosaccharide results in a positive fermentation test. Seven of the genes control the fermentation of carbohydrates by releasing fermentable hexose from an oligosaccharide. In Table 1 the genes and the substrates on which they act and some of the characteristics of the hypothetical enzymes are indicated. The vertical arrow indicates the point at which the molecule may be split.

\section{The gene $M Z$}

MZ controls the production of the adaptive enzyme, melezitase (Palleroni \& Lindegren, 1953). This gene exists in the form of a series of multiple alleles. Cells carrying the most 'complete' form of the gene are able to adapt to (and grow on) at least five different substrates: maltose, turanose, methyl- $\alpha-D-$ glucopyranoside, sucrose and melezitose. Growth on any one of these substrates produces adapted cells which are able to ferment any one of the other four and which can, in addition, split isomaltose and panose. Because of difficulty in obtaining sufficient amounts of the last two, it is not known whether there are MZ alleles conferring the capacity for growth in isomaltose and panose. One allele of $\mathbf{M Z}\left(\mathbf{M z}^{\mathrm{d}}\right)$ makes the cells capable of growth on maltose and turanose but incapable of growth on methyl- $\alpha$-D-glucopyranoside, sucrose or melezitose, and thus a culture carrying it superficially resembles one carrying MA mz. An ma MZd culture can be distinguished from an $\mathrm{Ma} \mathrm{mz}$ culture by transferring to melezitose (or sucrose, or methyl- $\alpha$-D-glucopyranoside) after growth on maltose: If the culture is ma $\mathbf{M z}^{\mathrm{d}}$, melezitose will be split; if it is MA mz, melezitose will not be split. The different alleles of MZ (Lindegren \& Lindegren, 1953) which have been identified are indicated in the following tabulation:

\begin{tabular}{|c|c|c|c|c|c|}
\hline 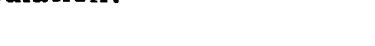 & & & enoty & & \\
\hline Substrate & MZ & MZa & $\mathbf{M} \mathbf{Z}^{\mathbf{b}}$ & $\mathbf{M Z} \mathbf{C}^{\mathbf{a}}$ & $\mathbf{M Z}^{\mathrm{d}}$ \\
\hline Maltose & + & + & + & + & + \\
\hline Turanose & + & + & + & + & + \\
\hline Sucrose & + & + & + & + & - \\
\hline Methyl- $\alpha$-D-glucopyranoside & + & - & + & - & - \\
\hline Melezitose & + & + & - & - & - \\
\hline Raffinose & - & - & - & - & - \\
\hline
\end{tabular}

Melezitase splits any terminal non-reducing $\alpha$-D-glucopyranosyl radical from a di- or trisaccharide, irrespective of whether it is attached to another hexose or simply to a methyl group and irrespective of the type of attachments to the hexose, except that it is not active against large molecules nor against a trisaccharide such as raffinose in which the non-reducing radical is blocked.

\section{The gene $M A$}

MA (Lindegren \& Lindegren, 1949) controls the production of an adaptive enzyme system which splits maltose and turanose. A culture carrying MA mz is distinguished from one carrying MA MZ or ma MZ by the fact that the 
웡 츨

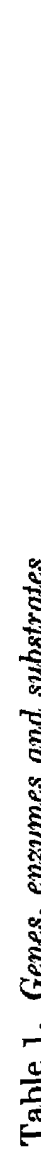

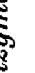

ญँ

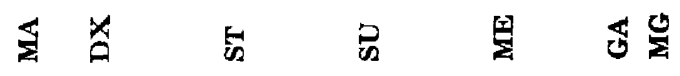

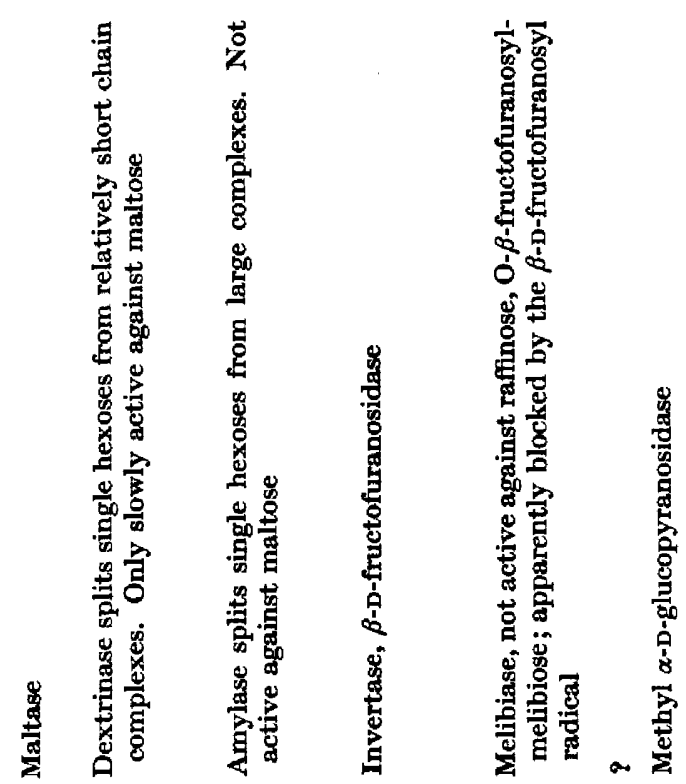

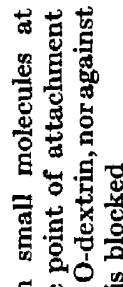

政

要

年

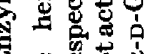

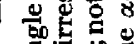

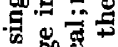

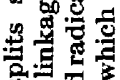

. 50

on

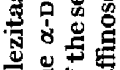

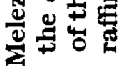

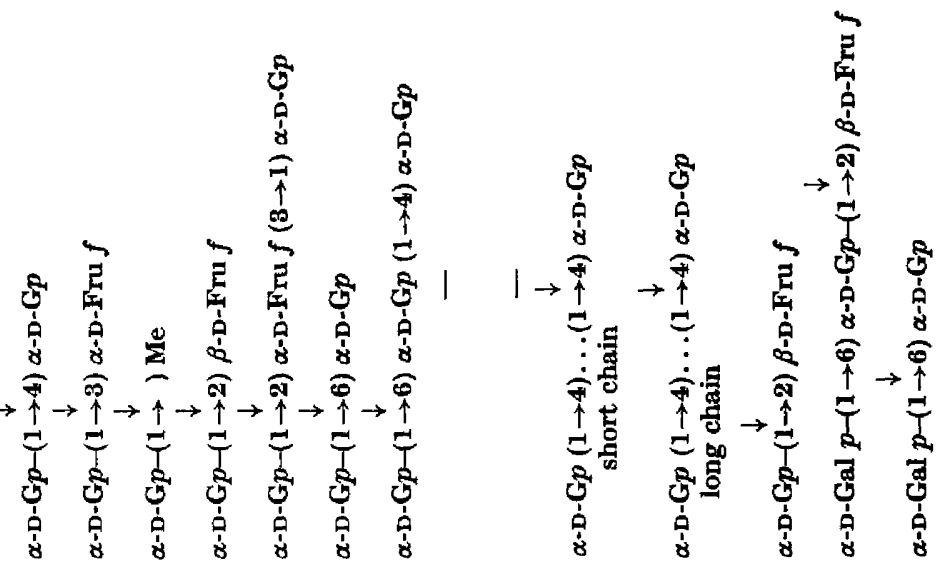

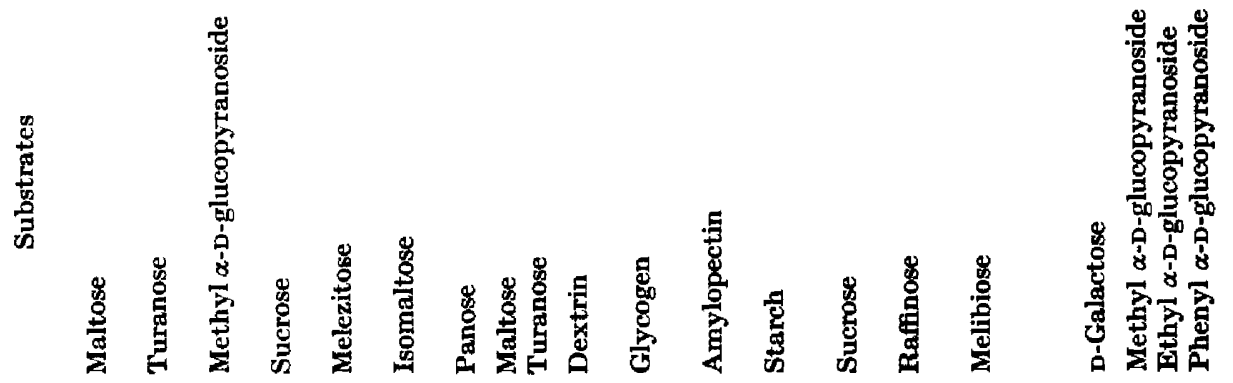


MA mz cultures do not achieve adaptation to melezitose by growth on maltose. Any culture which ferments maltose and turanose, and which (after growth on maltose) does not ferment melezitose, is MA mz.

Hestrin \& Lindegren $(1950,1952)$ found that an MA MG su mz culture when grown on maltose achieved the ability to ferment methyl- $\alpha$-D-glucopyranoside, but that an MA mg su mz culture was incapable of heterologous adaptation. This is the only case in the fermentation genotypes of detected interaction between two genes and is interpreted to indicate that the gene MG probably functions to produce a precursor of the maltase produced by MA. This inference is based on the fact that methyl- $\alpha$-D-glucopyranoside is very rare in nature and it seems improbable that a gene would be selected and perpetuated which produced an adaptive enzyme specific for it; it seems more probable that the gene MG functions primarily to enhance the activity of the MA gene.

\section{The gene $D X$}

DX (Lindegren, Lindegren, Drysdale, Hughes \& Brenes-Pomales, 1956) controls the synthesis of a dextrinase capable of splitting dextrin and glycogen but neither starch nor amylopectin. The failure of dextrinase to act against the substrates split by melezitose suggests that it splits off exposed reducing radicals; its slow action against maltose suggests that it is inhibited by the proximity of a non-reducing radical.

\section{The gene $S T$}

The gene ST produces an amylase which splits fermentable molecules from starch and amylopectin but not from either dextrin or glycogen.

The genes DX and ST originated from a culture of Saccharomyces diastaticus (Andrews \& Gilliland, 1952). Cultures which break down large carbohydrate polymers are exceedingly rare in Saccharomyces. The first Saccharomyces producing an enzyme of this type carried two such genes, and it is difficult to avoid the conclusion that one originated from the other. One parent of the pedigree (Tables 2 and 3 ) was a dextrin-fermenter originating from $S$. diastaticus. The other was descended from our inbred breeding stock. The hybrid was homozygous for the genes GA, mg and me; it was heterozygous for the genes indicated below :

\begin{tabular}{llllllllll}
\multicolumn{10}{c}{ Genotypes } \\
16119 & a & DX & ST & ma & SU & AN & HI & ur \\
\hline 16060 & a & dx & st & MA & su & an & hi & UR
\end{tabular}

The fermentative phenotypes are indicated below:

\begin{tabular}{llllllllll}
16119 & DXN & STA & MAL $^{\mathrm{L}}$ & tur & SUC & RAF & GAL & mgs & meb \\
\hline 16060 & dxn & sta & MAL & TUR & SUC $^{\mathrm{L}}$ & raf & GAL & mgs & meb
\end{tabular}




\section{Genes controlling carbohydrate fermentation}

Table 2. Pedigree of a hybrid heterozygous for $D X / d x, S T / s t$

\begin{tabular}{|c|c|c|c|c|c|c|c|c|}
\hline $\begin{array}{l}16201 \\
16202 \\
16203 \\
16204\end{array}$ & $\begin{array}{l}d \mathbf{x} \\
\mathbf{d x} \\
\mathbf{D X} \\
\mathbf{D X}\end{array}$ & $\begin{array}{l}\text { ST } \\
\text { st } \\
\text { st } \\
\text { ST }\end{array}$ & $\begin{array}{l}\text { TUR } \\
\text { tur } \\
\text { TUR } \\
\text { tur }\end{array}$ & $\begin{array}{l}\text { SU } \\
\text { su } \\
\text { su } \\
\text { SU }\end{array}$ & $\begin{array}{l}\mathbf{a} \\
\alpha \\
\alpha \\
\mathbf{a}\end{array}$ & $\begin{array}{l}\text { hi } \\
\text { HI } \\
\text { hi } \\
\text { HI }\end{array}$ & $\begin{array}{l}\text { an } \\
\text { AN } \\
\text { AN } \\
\text { an }\end{array}$ & \\
\hline $\begin{array}{l}16206 \\
16207\end{array}$ & $\begin{array}{l}\text { DX } \\
\text { Died }\end{array}$ & ST & TUR & su & $\alpha$ & hi & an & UR \\
\hline $\begin{array}{l}16208 \\
16200\end{array}$ & $\begin{array}{l}\mathrm{dx} \\
\mathrm{dx}\end{array}$ & $\begin{array}{l}\text { ST } \\
\text { st }\end{array}$ & $\begin{array}{l}\text { tur } \\
\text { TUR }\end{array}$ & $\begin{array}{l}\text { su } \\
\text { SU }\end{array}$ & $\begin{array}{l}\alpha \\
\mathbf{a}\end{array}$ & $\begin{array}{l}\text { hi } \\
\text { HI }\end{array}$ & $\begin{array}{l}\text { an } \\
\text { AN }\end{array}$ & $\begin{array}{l}\mathbf{u r} \\
\text { UR }\end{array}$ \\
\hline $\begin{array}{l}16210 \\
16211 \\
16212 \\
16213\end{array}$ & $\begin{array}{l}\mathrm{dx} \\
\mathrm{dx} \\
\mathrm{DX} \\
\mathbf{D X}\end{array}$ & $\begin{array}{l}\text { ST } \\
\text { st } \\
\text { st } \\
\text { ST }\end{array}$ & $\begin{array}{l}\text { tur } \\
\text { tur } \\
\text { TUR } \\
\text { TUR }\end{array}$ & $\begin{array}{l}\text { su } \\
\text { su } \\
\text { SU } \\
\text { SU }\end{array}$ & $\begin{array}{l}\alpha \\
\alpha \\
\mathbf{a} \\
\mathbf{a}\end{array}$ & $\begin{array}{l}\text { hi } \\
\text { hi } \\
\text { HI } \\
\text { HI }\end{array}$ & $\begin{array}{l}\text { AN } \\
\text { AN } \\
\text { an } \\
\text { an }\end{array}$ & $\begin{array}{l}\text { ur } \\
\text { UR } \\
\text { UR } \\
\text { ur }\end{array}$ \\
\hline $\begin{array}{l}16222 \\
16223 \\
16224 \\
16225\end{array}$ & $\begin{array}{l}\mathbf{D X} \\
\mathbf{d x} \\
\mathbf{d x} \\
\mathbf{D X}\end{array}$ & $\begin{array}{l}\text { st } \\
\text { ST } \\
\text { ST? } \\
\text { ST }\end{array}$ & $\begin{array}{l}\text { TUR } \\
\text { tur } \\
\text { TUR } \\
\text { tur }\end{array}$ & $\begin{array}{l}\text { su } \\
\text { su } \\
\text { SU } \\
\text { SU }\end{array}$ & $\begin{array}{l}\alpha \\
\mathbf{a} \\
\mathbf{a} \\
\alpha\end{array}$ & $\begin{array}{l}\text { HI } \\
\text { HI } \\
\text { hi } \\
\text { hi }\end{array}$ & $\begin{array}{l}\text { AN } \\
\text { AN } \\
\text { an } \\
\text { an }\end{array}$ & $\begin{array}{l}\text { ur } \\
\text { UR } \\
\text { UR } \\
\text { ur }\end{array}$ \\
\hline $\begin{array}{l}16226 \\
16227 \\
16228 \\
16229\end{array}$ & $\begin{array}{l}\mathrm{dx} \\
\mathrm{DX} \\
\mathrm{dx} \\
\mathrm{DX}\end{array}$ & $\begin{array}{l}\text { ST? } \\
\text { st } \\
\text { st } \\
\text { ST? }\end{array}$ & $\begin{array}{l}\text { TUR } \\
\text { TUR } \\
\text { tur } \\
\text { tur }\end{array}$ & $\begin{array}{l}\text { SU } \\
\text { su } \\
\text { SU } \\
\text { su }\end{array}$ & $\begin{array}{l}a \\
\alpha \\
\alpha \\
?\end{array}$ & $\begin{array}{l}\text { hi } \\
\text { hi } \\
\text { hi } \\
\text { HI }\end{array}$ & $\begin{array}{l}\text { AN } \\
\text { an } \\
\text { an } \\
\text { AN }\end{array}$ & $\begin{array}{l}\text { UR } \\
\text { UR } \\
\text { ur } \\
\text { ur } \\
\text { ur }\end{array}$ \\
\hline $\begin{array}{l}16242 \\
16243 \\
16244 \\
16245\end{array}$ & $\begin{array}{l}\mathrm{dx} \\
\mathrm{DX} \\
\mathrm{dx} \\
\mathrm{Dx}\end{array}$ & $\begin{array}{l}\text { ST } \\
\text { st } \\
\text { ST } \\
\text { ST }\end{array}$ & $\begin{array}{l}\text { TUR } \\
\text { tur } \\
\text { tur } \\
\text { TUR }\end{array}$ & $\begin{array}{l}\text { SU } \\
\text { su } \\
\text { SU } \\
\text { su }\end{array}$ & $\begin{array}{l}a \\
a \\
\alpha \\
\alpha\end{array}$ & $\begin{array}{l}\text { HI } \\
\text { hi } \\
\text { hi } \\
\text { HI }\end{array}$ & $\begin{array}{l}\text { AN } \\
\text { an } \\
\text { an } \\
\text { AN }\end{array}$ & $\begin{array}{l}\text { ur } \\
\text { UR } \\
\text { UR } \\
\text { UR }\end{array}$ \\
\hline $\begin{array}{l}16246 \\
16247 \\
16248 \\
16249\end{array}$ & $\begin{array}{l}\text { DX } \\
\mathbf{d x} \\
\text { Died } \\
d \mathbf{d x}\end{array}$ & $\begin{array}{l}\text { st } \\
\text { st }\end{array}$ & $\begin{array}{l}\text { tur } \\
\text { TUR } \\
\text { TUR }\end{array}$ & $\begin{array}{l}\text { SU } \\
\text { SU }\end{array}$ & $\begin{array}{l}\alpha \\
\text { a? } \\
\alpha\end{array}$ & $\begin{array}{l}\text { HI } \\
\text { HI } \\
\text { hi }\end{array}$ & $\begin{array}{l}\text { AN } \\
\mathbf{A N} \\
\text { an }\end{array}$ & $\begin{array}{l}\mathbf{u r} \\
\mathbf{U R} \\
\mathbf{u r}\end{array}$ \\
\hline $\begin{array}{l}16251 \\
16252 \\
16253 \\
16254\end{array}$ & $\begin{array}{l}\mathrm{DX} \\
\mathrm{dx} \\
\mathrm{dx} \\
\mathrm{Dx}\end{array}$ & $\begin{array}{l}\text { st } \\
\text { st } \\
\text { st } \\
\text { st }\end{array}$ & $\begin{array}{l}\text { TUR } \\
\text { TUR } \\
\text { tur } \\
\text { tur }\end{array}$ & $\begin{array}{l}\text { su } \\
\text { sU } \\
\text { su } \\
\text { SU }\end{array}$ & $\begin{array}{l}\mathrm{a} \\
\alpha \\
\mathrm{a} q \\
\alpha\end{array}$ & $\begin{array}{l}\text { HI } \\
\text { hi } \\
\text { HI } \\
\text { hi }\end{array}$ & $\begin{array}{l}\text { AN } \\
\text { an } \\
\text { AN } \\
\text { an }\end{array}$ & $\begin{array}{l}\text { ur } \\
\text { UR } \\
\text { UR } \\
\text {. }\end{array}$ \\
\hline $\begin{array}{l}16255 \\
16256 \\
16257 \\
16258\end{array}$ & $\begin{array}{l}\mathrm{DX} \\
\mathrm{dx} \\
\mathrm{DX} \\
\mathrm{dx}\end{array}$ & $\begin{array}{l}\text { st } \\
\text { st } \\
\text { st } \\
\text { st }\end{array}$ & $\begin{array}{l}\text { tur } \\
\text { tur } \\
\text { TUR } \\
\text { TUR }\end{array}$ & $\begin{array}{l}\text { su } \\
\text { SU } \\
\text { su } \\
\text { SU }\end{array}$ & $\begin{array}{l}\alpha \\
\alpha \\
\text { a? } \\
a\end{array}$ & $\begin{array}{l}\text { HI } \\
\text { hi } \\
\text { hi } \\
\text { HI }\end{array}$ & $\begin{array}{l}\text { AN } \\
\text { an } \\
\text { an } \\
\text { AN }\end{array}$ & $\begin{array}{l}\mathbf{u r} \\
\mathbf{u r} \\
\mathbf{U R} \\
\mathbf{U R}\end{array}$ \\
\hline $\begin{array}{l}16259 \\
16260 \\
16261 \\
16262\end{array}$ & $\begin{array}{l}\mathrm{dx} \\
\mathbf{D X} \\
\mathbf{D X} \\
\mathrm{dx}\end{array}$ & $\begin{array}{l}\text { st } \\
\text { st } \\
\text { ST } \\
\text { st }\end{array}$ & $\begin{array}{l}\text { TUR } \\
\text { TUR } \\
\text { tur } \\
\text { tur }\end{array}$ & $\begin{array}{l}\text { SU } \\
\text { su } \\
\text { SU } \\
\text { su }\end{array}$ & $\begin{array}{l}\text { a? } \\
a \\
\alpha \\
\alpha\end{array}$ & $\begin{array}{l}\text { HI } \\
\text { hi } \\
\text { HI } \\
\text { hi }\end{array}$ & $\begin{array}{l}\text { an } \\
\text { AN } \\
\text { AN } \\
\text { an }\end{array}$ & $\begin{array}{l}\text { ur } \\
\text { UR } \\
\text { ur } \\
\text { UR }\end{array}$ \\
\hline $\begin{array}{l}16267 \\
16268 \\
16269 \\
16270\end{array}$ & $\begin{array}{l}\mathrm{dx} \\
\mathrm{DX} \\
\mathrm{dx} \\
\mathrm{DX}\end{array}$ & $\begin{array}{l}\text { ST } \\
\text { ST } \\
\text { st } \\
\text { st }\end{array}$ & $\begin{array}{l}\text { tur } \\
\text { TUR } \\
\text { TUR } \\
\text { tur }\end{array}$ & $\begin{array}{l}\text { su } \\
\text { SU } \\
\text { su } \\
\text { SU }\end{array}$ & $\begin{array}{l}\mathrm{a} ? \\
\alpha \\
\alpha \\
\mathbf{a}\end{array}$ & $\begin{array}{l}\text { hi } \\
\text { HI } \\
\text { hi } \\
\text { HI }\end{array}$ & $\begin{array}{l}\text { AN } \\
\text { AN } \\
\text { an } \\
\text { an }\end{array}$ & $\begin{array}{l}\text { ur } \\
\text { UR } \\
\text { UR } \\
\text { ur }\end{array}$ \\
\hline $\begin{array}{l}16271 \\
16272 \\
16273\end{array}$ & $\begin{array}{l}\mathrm{dx} \\
\mathbf{D x} \\
\mathrm{dx}\end{array}$ & $\begin{array}{l}\text { st } \\
\text { ST } \\
\text { ST }\end{array}$ & $\begin{array}{l}\stackrel{\cdot}{\text { tur }} \\
\text { TUR }\end{array}$ & $\begin{array}{l}\text { su } \\
\text { SU } \\
\text { SU }\end{array}$ & $\begin{array}{l}\alpha \\
\mathbf{a} \\
\mathbf{a}\end{array}$ & $\begin{array}{l}\text { HI } \\
\text { HI } \\
\text { hi }\end{array}$ & $\begin{array}{l}\text { AN } \\
\text { AN } \\
\text { an }\end{array}$ & $\begin{array}{l}\text { UR } \\
\text { ur } \\
\text { ur }\end{array}$ \\
\hline $\begin{array}{l}16274 \\
16275\end{array}$ & $\dot{\text { Died }}$ & . & . & . & . & • & . & UR \\
\hline $\begin{array}{l}16276 \\
16277 \\
16278\end{array}$ & $\begin{array}{l}\mathrm{dx} \\
\mathrm{dx} \\
\mathrm{DX}\end{array}$ & $\begin{array}{l}\text { st } \\
\text { st } \\
\text { st }\end{array}$ & . & : & $\dot{.}$ & $\dot{.}$ & . & $\begin{array}{l}\text { ur } \\
\mathbf{U R} \\
\mathbf{u r}\end{array}$ \\
\hline $\begin{array}{l}16299 \\
16300 \\
16301 \\
16302\end{array}$ & $\begin{array}{l}\mathrm{dx} \\
\mathrm{Dx} \\
\mathrm{dx} \\
\mathrm{Dx}\end{array}$ & $\begin{array}{l}\text { ST? } \\
\text { st } \\
\text { st } \\
\text { st }\end{array}$ & $\begin{array}{l}\text { TUR } \\
\text { tur } \\
\text { TUR } \\
\text { tur }\end{array}$ & $\begin{array}{l}\text { su } \\
\text { SU } \\
\text { SU } \\
\text { su }\end{array}$ & $\begin{array}{l}a \\
\alpha \\
a \\
\alpha\end{array}$ & $\begin{array}{l}\text { hi } \\
\text { hi } \\
\text { hi } \\
\text { HI }\end{array}$ & $\begin{array}{l}\text { an } \\
\text { an } \\
\text { an } \\
\mathrm{AN}\end{array}$ & $\begin{array}{l}\text { ur } \\
\text { UR } \\
\text { ur } \\
\text { ur }\end{array}$ \\
\hline
\end{tabular}


The abbreviations listing genotype first are DX, DXN-dextrin; ST, STAstarch; MA, MAL-maltose; TUR-turanose; SUC-sucrose; RAF-raffinose; AN-anthranilic acid; HI-histidine; UR-uracil; GA, GAL-galactose; MG, MGS-methyl- $\alpha$-D-glucopyranoside; ME, MEB-melibiose.

16119 was turanose-negative and therefore ma. It is characteristic of cultures carrying the gene DX to produce gas slowly from maltose. This may be due to the presence of small amounts of dextrin in the maltose.

Genetical separation of dextrinase and amylase. Regular segregation of genes $\mathrm{DX} / \mathrm{dx}$ occurred in every ascus. Although this was not true of $\mathrm{ST} / \mathrm{st}$, it is clear that DX and ST segregate independently. Since most of the cultures fermented maltose, the diagnosis for MA was made by action on turanose. Regular segregation of the other genes indicated in the pedigree show that a true hybrid was produced and that the failure of the S'T gene to segregate regularly was not due to polyploidy. In Table 3 detailed analysis of the diagnosis of the genes DX and ST is given. The same fourteen asci were analysed, using the melting-point tube technique. The tubes were discarded after 7 days, and if fermentation had not occurred up to that time were registered as negatives and indicated by the minus sign.

\section{Table 3. Pedigree showing fermentation of carbohydrate polymers}

Each minus sign indicates a separate test. Two series of tests were made and the results are listed under $\mathbf{A}$ and $\mathbf{B}$, respectively. The day on which fermentation occurred is indicated by Arabic numerals and if more than one positive fermentation occurred more than one numeral is listed. For instance, culture 16208 is listed as a positive fermenter of dextrin as indicated by the numerals 333. This means that three tests were made and all proved positive on the third day.

\begin{tabular}{|c|c|c|c|c|c|c|c|c|c|c|}
\hline & \multicolumn{2}{|c|}{ Dextrin } & \multicolumn{2}{|c|}{ Glycogen } & \multicolumn{2}{|c|}{ Starch } & \multicolumn{2}{|c|}{ Amylopectin } & \multicolumn{2}{|c|}{$\begin{array}{c}\text { Schardinger } \\
\text { dextrin }\end{array}$} \\
\hline & $\mathbf{A}$ & $\mathbf{B}$ & $\mathbf{A}$ & $\mathbf{B}$ & $\mathbf{A}$ & $\mathbf{B}$ & $\mathbf{A}$ & $\mathbf{B}$ & $\mathbf{A}$ & B \\
\hline 16201 & $\equiv$ & $=$ & $\equiv$ & $=$ & $5-$ & $=$ & $=$ & $=$ & $=$ & $=$ \\
\hline 16202 & $\equiv$ & $=$ & $\equiv$ & $=$ & $=$ & $=$ & 4 & $=$ & $=$ & $=$ \\
\hline 16203 & 333 & 11 & $\equiv$ & $=$ & $=$ & $=$ & $=$ & $=$ & $=$ & $=$ \\
\hline 16204 & 338 & 11 & 333 & 55 & $5-$ & $=$ & $=$ & $=$ & $5-$ & $=$ \\
\hline $\begin{array}{l}16206 \\
16207\end{array}$ & $\begin{array}{l}333 \\
\text { Died }\end{array}$ & 11 & 555 & 23 & $5-$ & 33 & $=$ & $=$ & $=$ & $=$ \\
\hline 16208 & $=$ & $=$ & $=$ & $=$ & $5-$ & - & 44 & $=$ & $=$ & $=$ \\
\hline 16209 & $=$ & $=$ & $=$ & $=$ & $=$ & 45 & $=$ & $=$ & $=$ & $=$ \\
\hline 16210 & - & $=$ & $\equiv$ & $=$ & $\mathbf{5}-$ & $=$ & $5-$ & $=$ & 55 & $=$ \\
\hline 16211 & $\equiv$ & $=$ & $\equiv$ & $=$ & $=$ & $=$ & $=$ & $=$ & $=$ & $=$ \\
\hline 16212 & 333 & 22 & $77-$ & $=$ & $=$ & $=$ & $=$ & $=$ & $=$ & $=$ \\
\hline 10213 & 333 & 22 & 555 & $=$ & $5-$ & $=$ & $4-$ & $=$ & $=$ & $=$ \\
\hline 16222 & . & . & . & . & . & . & . & . & . & . \\
\hline 16223 & $\equiv$ & $=$ & $\equiv$ & $=$ & $5-$ & $4-$ & $=$ & $=$ & $=$ & $=$ \\
\hline 16224 & $=$ & $=$ & $=$ & $=$ & $=$ & 4- & $=$ & $=$ & $=$ & $=$ \\
\hline 16225 & 333 & 11 & $\mathbf{3 3 3}$ & 55 & $5-$ & 4 & $=$ & $=$ & $=$ & $=$ \\
\hline 16226 & $\equiv$ & $=$ & $\equiv$ & $=$ & $\equiv$ & $=$ & $=$ & $5-$ & $=$ & $=$ \\
\hline 16227 & 338 & 11 & $66-$ & 66 & $=$ & $=$ & $=$ & $=$ & $=$ & $=$ \\
\hline 10228 & $\equiv$ & $=$ & $=$ & $=$ & $=$ & $=$ & $=$ & $=$ & $=$ & $=$ \\
\hline 16229 & 338 & 11 & 444 & 55 & $=$ & $4-$ & $=$ & $=$ & $=$ & $=$ \\
\hline
\end{tabular}


Table 3 (cont.)

\begin{tabular}{|c|c|c|c|c|c|c|c|c|c|c|}
\hline & Dex & & Glyc & & Star & & Amy & ectin & $\begin{array}{r}\text { Sche } \\
\text { de }\end{array}$ & $\begin{array}{l}\text { nger } \\
\text { in }\end{array}$ \\
\hline & A & B & $\mathbf{A}$ & B & $\mathbf{A}$ & B & $\mathbf{A}$ & B & $\mathbf{A}$ & B \\
\hline 16242 & $\equiv$ & $=$ & $\equiv$ & $=$ & 77 & $=$ & $=$ & $3 ?$ & $=$ & $=$ \\
\hline 16243 & 333 & 33 & 666 & $=$ & $=$ & $=$ & $=$ & $=$ & $=$ & $=$ \\
\hline 16244 & $\equiv$ & $=$ & $\equiv$ & $=$ & $=$ & 47 & $=$ & $=$ & $=$ & $=$ \\
\hline 16245 & 222 & 11 & $\mathbf{3 3}$ & 22 & $5-$ & $=$ & $=$ & $=$ & $=$ & $=$ \\
\hline 16246 & 22 & 11 & 66 & 44 & $=$ & $=$ & $=$ & $=$ & $=$ & $=$ \\
\hline 16247 & $=$ & $=$ & $=$ & $=$ & $=$ & $=$ & $=$ & $=$ & $=$ & $=$ \\
\hline 16248 & Died & & & & & & & & & \\
\hline 16249 & $=$ & $=$ & $=$ & $=$ & $=$ & $=$ & $=$ & $=$ & $=$ & $=$ \\
\hline 16251 & 333 & 11 & 383 & 44 & $=$ & $=$ & $=$ & $=$ & $=$ & $=$ \\
\hline 16252 & $=$ & $=$ & $=$ & $=$ & $=$ & $=$ & $=$ & $=$ & $=$ & $=$ \\
\hline 16253 & $=$ & $=$ & $=$ & $=$ & $=$ & $=$ & $=$ & $=$ & $=$ & $=$ \\
\hline 16254 & 333 & 11 & 383 & 77 & $=$ & $=$ & $=$ & $=$ & $=$ & $=$ \\
\hline 16255 & . & 11 & . & 83 & . & . & . & $=$ & . & $=$ \\
\hline 16256 & . & & . & . & . & . & . & . & . & . \\
\hline 16257 & $11=$ & 11 & $55=$ & 22 & . & $=$ & . & $=$ & $=$ & $=$ \\
\hline 16258 & $=$ & $=$ & $=$ & $=$ & $=$ & $=$ & $=$ & $=$ & $=$ & $=$ \\
\hline 16259 & $6-$ & $=$ & $=$ & $=$ & $=$ & $=$ & $=$ & $=$ & $=$ & $=$ \\
\hline 16260 & 33 & 11 & 38 & 55 & $=$ & $=$ & $=$ & $=$ & $=$ & $=$ \\
\hline 16261 & 11 & 44 & 444 & $=$ & $=$ & 45 & $=$ & 44 & $=$ & $=$ \\
\hline 16262 & $=$ & $=$ & . & $=$ & . & $=$ & . & . & . & . \\
\hline 16267 & $\equiv$ & $=$ & $\equiv$ & $=$ & 55 & 5 & $=$ & 5 & $=$ & $=$ \\
\hline 16268 & 333 & 11 & 333 & 44 & 55 & $5-$ & 44 & 4- & $=$ & $=$ \\
\hline 16269 & 5 & $=$ & $=$ & $\mathbf{3}$ & $=$ & $=$ & $=$ & $=$ & $=$ & $=$ \\
\hline 16270 & $\mathbf{3 3 3}$ & 11 & $\equiv$ & 22 & $\Rightarrow$ & $=$ & $=$ & $=$ & $=$ & $=$ \\
\hline 16271 & $\equiv$ & $=$ & $\equiv$ & $=$ & . & $=$ & . & $=$ & . & $=$ \\
\hline 16272 & 44 & 23 & $=$ & $=$ & $=$ & 66 & $=$ & 2 & $=$ & $=$ \\
\hline 16273 & $=$ & $=$ & $=$ & $=$ & $=$ & 77 & 55 & $=$ & $=$ & $=$ \\
\hline 16274 & . & $=$ & . & $=$ & . & $=$ & . & $=$ & . & $=$ \\
\hline 16275 & Died & & & & & & & & & \\
\hline 16276 & $\equiv$ & $=$ & $\equiv$ & $=$ & $=$ & $=$ & $=$ & $=$ & $=$ & $=$ \\
\hline 16277 & $=$ & $=$ & $=$ & $=$ & . & $=$ & . & $=$ & . & $=$ \\
\hline 16278 & 44 & 44 & 777 & 55 & $=$ & $=$ & $=$ & $=$ & $=$ & $=$ \\
\hline 16299 & $=$ & $=$ & $=$ & $=$ & $=$ & $=$ & $=$ & 66 & $=$ & $=$ \\
\hline 16300 & 11 & 11 & 22 & 3 & $=$ & $=$ & $=$ & $=$ & $=$ & $=$ \\
\hline 16301 & $=$ & $=$ & $=$ & $=$ & $=$ & $=$ & $=$ & $=$ & $=$ & $=$ \\
\hline 16302 & 11 & 11 & 22 & 22 & $=$ & $=$ & $=$ & $=$ & $=$ & $=$ \\
\hline
\end{tabular}

Dextrin ferments more readily than the other large polymers, occasionally showing a positive fermentation on the first day and generally showing a positive fermentation on the third day. Glycogen ferments less rapidly, no positives occurring on the first day. Whole cell preparations were used and the possibility that the same enzyme was involved in both fermentations is not excluded. A series of tests on starch and amylopectin revealed a general correspondence between the fermentability of these two substrates and indicated that dextrin-positive, glycogen-positive cultures were occasionally starch- and amylopectin-negative and, conversely, that some starch-and amylopectin-positive cultures were occasionally dextrin- and glycogennegative. Two positive tests were obtained with Schardinger dextrin; one 
of the Schardinger positives was dextrin-positive and the other dextrinnegative. These data indicate that dextrin is a reliable and precise indicator of the presence of the gene DX. The fermentation of starch and amylopectin is either so difficult or the expressiveness of the gene is so restricted that unless some special purpose can be served by analysing for this genotype, it will not be particularly helpful in mapping experiments. There are some indications that the enzymes produced by DX and ST are constitutive.

The tetratype ascus 16201-16204 produced a culture capable of fermenting both dextrin and starch (DX ST), one capable of fermenting dextrin but not starch (DX st), one incapable of fermenting dextrin but capable of fermenting starch (dx ST) and one incapable of fermenting either starch or dextrin (dx st). The pedigree was completely regular with regard to the segregation of ability to ferment dextrin; two fermenters and two non-fermenters were found in every ascus. The ability to ferment starch was irregularly segregated, some tetrads producing more than two fermenters per ascus and others not producing any fermenters. The ability to ferment turanose is the diagnostic criterion for the gene MA. It is clear that a starch fermenter in a regular tetrad such as 16201-16204 may be turanose-negative and, therefore, ma. Similarly, a dextrin-fermenter in a regular ascus such as 16201-16204 may be turanosenegative and, therefore, ma. Although these data clearly indicate the independence of the genes controlling the fermentation of starch and dextrin, they also suggest that there are diagnostic difficulties involved in analysing hybrids heterozygous for the ability to ferment starch. Amylase probably splits single hexoses from large $\mathrm{D}$-glucan molecules at the 1,4 linkage. If primary action is on 1, 4 linkages, action on 1, 6 linkages seems unlikely since the 1, 6 linkages are buried inside the polysaccharide molecule. The gene DX does not confer the ability to ferment turanose. Any dextrin-positive culture which is turanosenegative is DX ma, although it may act slowly on maltose.

Five dxn STA tur cultures (serial numbers 16208, 16210, 16223, 16244 and 16267) were studied critically for their capacity to ferment maltose and found to be maltose-negative. This observation reveals that starch-positive cultures may be completely incapable of acting on maltose, and that possession of the gene ST does not confer the ability to split maltose. It appears that starch is not disintegrated by breaking off single maltose molecules but presumably by splitting off single hexose molecules. It has been suggested that DX produces an enzyme which acts slowly on maltose because it may be inhibited by the proximity of a non-reducing radical. The complete absence of any action by the gene ST on maltose suggests that the same restriction applies to a much greater degree to the enzyme produced by ST and that it is able to split off terminal reducing radicals of starch only because they are so far removed from the non-reducing radicals. The experiment was controlled by testing the ability of the dxn STA TUR cultures 16299,16273 and 16242 to act on maltose. They fermented maltose rapidly. Cultures 16249 and 16258 , which are dxn sta TUR, were also rapid fermenters of maltose. 


\section{The gene $S U$}

SU controls the production of a constitutive enzyme, $\beta$-D-fructofuranosidase (invertase) which splits the exposed $\beta$-D-fructofuranosyl radical from either sucrose or raffinose. Any culture fermenting raffinose under standard conditions is SU. Raffinose is the unique substrate which identifies SU; the enzyme produced by $\mathrm{MZ}$ also splits sucrose but is incapable of splitting raffinose.

\section{The gene $M E$}

ME controls the production of an adaptive enzyme splitting melibiose but not raffinose. Any melibiose-positive culture is identified as ME (Lindegren, 1949). Melibiase splits the terminal $\alpha$-D-glucopyranosyl radical from melibiose. It is blocked from acting on raffinose by the $\beta$-D-fructofuranosyl radical.

\section{The gene $M G$}

MG (Lindegren \& Lindegren, 1949) controls the production of an adaptive enzyme methyl- $\alpha$-D-glucopyranosidase; some data indicate that both phenyland ethyl- $\alpha$-D-glucopyranoside are also split; the enzyme is capable of splitting non-hexose radicals from substituted $\alpha$-D-glucopyranosides but not from disaccharides. Any maltose-negative culture which is methyl- $\alpha$-D-glucopyranoside-positive is identified as MG.

We are indebted to Dr Maurice Ogur for many suggestions concerning the modes of action of the gene-controlled enzymes. We have corresponded with many carbohydrate chemists and enzymologists and wish to acknowledge their helpful suggestions but hesitate to name them directly to avoid the implication that they are in agreement with the inferences made. We are indebted to Dr Allene Jeanes, Dr N. K. Richtmyer, Dr E. J. Hehre, Dr M. L. Killey, Dr R. J. Dimler, Dr W. W. Pigman and to E. R. Squibb and Sons for gifts of rare sugars.

This work has been supported by research grants from the National Cancer Institute of the National Institutes of Health, Public Health Service C-1179 and the U.S. Atomic Energy Commission AT (11-1) 176.

\section{REFERENCES}

ANDrews, J. \& GilliLAND, R. B. (1952). Super-attenuation of beer: a study of three organisms capable of causing abnormal attenuations. J. Inst. Brew. 58, 189.

Cardini, C. E., Caputto, R., Paladini, A. C. \& Leloir, L. F. (1950). Uridine diphosphate glucose: the coenzyme of the galactose-glucose phosphate isomerization. Nature, Lond. 165, 191.

Hestrin, S. \& Lindegren, C. C. (1950). Carbohydrases in Saccharomyces haploid stocks of defined genotype. I. Fermentation and hydrolysis of $\alpha$-glucosides by yeast 6233. Arch. Biochem. 29, 315.

Hestrin, S. \& Lindegren, C. C. (1952). Carbohydrases in Saccharomyces. II. Genecontrolled induction of glucosidases by $\alpha$-glucosides. Arch. Biochem. Biophys. $38,317$.

Lindegren, C. C. (1949). The Yeast Cell, Its Genetics and Cytology. St. Louis, Mo.: Educational Publishers, Inc. 
Lindegren, C. C. (1956). Methods for the diagnosis of gene-controlled fermentative ability in Saccharomyces. Wallerstein Labs. Commun. 19, 49.

Lindegren, C. C. \& Lindegren, G. (1946). The cytogene theory. Cold Spr. Harb. Symp. quant. Biol. 11, 115.

LiNDEgREN, C. C. \& Lindegren, G. (1949). Unusual gene-controlled combinations or carbohydrate fermentations in yeast hybrids. Proc. nat. Acad. Sci., Wash. 35, 23.

Lindegren, C. C. \& Lindegren, G. (1953). The genetics of melezitose fermentation in Saccharomyces. Genetica, 26, 430.

Lindegren, C. C., Lindegren, G., Drysdale, R. B., Hughes, J. P. \& BrenesPomales, A. (1956). Genetical analysis of the clones from a single tetrad of Saccharomyces showing non-Mendelian segregation. Genetica (in the Press).

Palleroni, N. J. \& Lindegren, C. C. (1953). A single adaptive enzyme in Saccharomyces elicited by several related substrates. J. Bact. 65, 122.

(Received 15 September 1955) 